\title{
Backfolded acoustic phonons as ultrasonic probes in metal-oxide superlattices
}

\author{
F. Lyzwa, ${ }^{1}$ A. Chan, ${ }^{2,3}$ J. Khmaladze $\odot,{ }^{1}$ K. Fürsich $\odot,{ }^{4}$ B. Keimer ${ }^{4}$ C. Bernhard, ${ }^{1}$ M. Minola $\odot,{ }^{4}$ and B. P. P. Mallett $\odot^{2,5,}, *$ \\ ${ }^{1}$ Department of Physics and Fribourg Center for Nanomaterials, University of Fribourg, Chemin du Musée 3, CH-1700 Fribourg, Switzerland \\ ${ }^{2}$ The MacDiarmid Institute for Advanced Materials and Nanotechnology and The Dodd Walls Centre for Quantum \\ and Photonic Technologies, 1010 Auckland, New Zealand \\ ${ }^{3}$ School of Chemical Sciences, The University of Auckland, 1010 Auckland, New Zealand \\ ${ }^{4}$ Max-Planck-Institut für Festkörperforschung, Heisenbergstrasse 1, 70569 Stuttgart, Germany \\ ${ }^{5}$ Department of Physics, The University of Auckland, 1010 Auckland, New Zealand
}

(Received 19 January 2020; accepted 25 March 2020; published 28 April 2020)

\begin{abstract}
Ultrasonics have been an incisive probe of internal interfaces in a wide variety of systems ranging from stars to solids. For thin-film structures, however, ultrasound is largely ineffective because the signal is dominated by the substrate. Using confocal Raman spectromicroscopy, we show that multiple reflection of sound waves at internal interfaces of a metal-oxide superlattice generates standing waves that are insensitive to the substrate. Such modes had previously been observed only in high-quality superlattices of elemental semiconductors, and their observation in complex metal-oxide heterostructures is testimony to recent progress in this field. We use the high spatial resolution of the Raman microscope to demonstrate the high sensitivity of the mode frequency to atomic-scale thickness variations of the superlattice. Spectroscopy of acoustic standing waves can hence serve as a powerful characterization tool of thin-film structures. In analogy to ultrasound spectroscopy of bulk solids, lineshape analysis of these modes has the potential to yield detailed information about the internal structure of the interfaces as well as the coupling of sound waves to the low-frequency spin, charge, and orbital dynamics in metal-oxide superlattices.
\end{abstract}

DOI: 10.1103/PhysRevMaterials.4.043606

\section{INTRODUCTION}

Thin-film multilayers of metal oxides host a wide range of emergent, tunable, and potentially useful properties [1-10] beyond those observed in multilayers of more conventional semiconductors such as $\mathrm{Si}$ and GaAs $[11,12]$. This is due to the various types of magnetic, charge, ferroelectric, and superconducting orders that the constituent metal-oxide layers can host $[13,14]$. The novel properties of thin-film multilayers and superlattices result from interactions across the interface, such as spin and orbital reconstruction, charge transfer, and phonon coupling [3-5,7], as well as some hitherto unidentified mechanisms $[9,15]$. The additional periodicity introduced in superlattice structures with smooth interfaces can also be used to tune the thermal conductivity via the opening of phononic band gaps [16]. As such, multilayers of metal oxides constitute a fertile playground both to discover interesting physics and to tailor functionalities that could shape future electronics and phononics [16,17].

Besides the scientific challenge of understanding the properties of metal-oxide superlattices, there is the persistent technical challenge of maintaining the quality of the crystal structure, interfaces, and layer thickness in such samples [18]. Growth techniques for such samples include [18] pulsed laser deposition (PLD) [19], molecular beam epitaxy [20], and sputtering [21].

\footnotetext{
*benjamin.mallett@gmail.com
}

Here we report on the observation of low-frequency Raman modes in metal-oxide superlattices, with a focus on superlattices of the high-temperature superconductor $\mathrm{YBa}_{2} \mathrm{Cu}_{3} \mathrm{O}_{7}$ (YBCO) and $R \mathrm{MnO}_{3}$ manganites, which are exemplary multilayers for displaying the effects described above [7,9,15]. The low-energy modes are optical phonons which arise in superlattices due to the backfolding of the $c$-axis acoustic phonon branch and, to the best of our knowledge, are detected for the first time in metal-oxide heterostructures. Until now the observation of backfolded acoustic phonon modes has only been reported in superlattices of high-quality molecular-beam-epitaxy-grown semiconductor superlattices, such as GaAs/AlAs [22-26]. The occurrence and the properties of these modes were fully explained and described within a comprehensive theoretical framework [24,27-29]. The modes can thus be used to characterize the quality and properties of the superlattice, such as the speed of sound and the bilayer thickness, and signify the opening of a small phononic bandgap [17]. Given the fast acquisition time of the measurement and the potential for micrometer spatial resolution, such modes can be a useful diagnostic for characterizing superlattices of given materials.

\section{METHODS}

In the present work we study epitaxial superlattices of $\mathrm{YBa}_{2} \mathrm{Cu}_{3} \mathrm{O}_{7}$ and manganite compositions $R \mathrm{MnO}_{3}$, with $R=$ $\mathrm{Pr}_{0.5} \mathrm{La}_{0.2} \mathrm{Ca}_{0.3}$ (PLCMO), $\mathrm{Nd}_{1-x}\left(\mathrm{Ca}_{1-y} \mathrm{Sr}_{y}\right)_{x}$ (NCSMO), or $\mathrm{La}_{1-x} \mathrm{Ca}_{x}$ (LCMO). Details of the sample compositions can be found in the Supplementary Material [30]. The samples are 


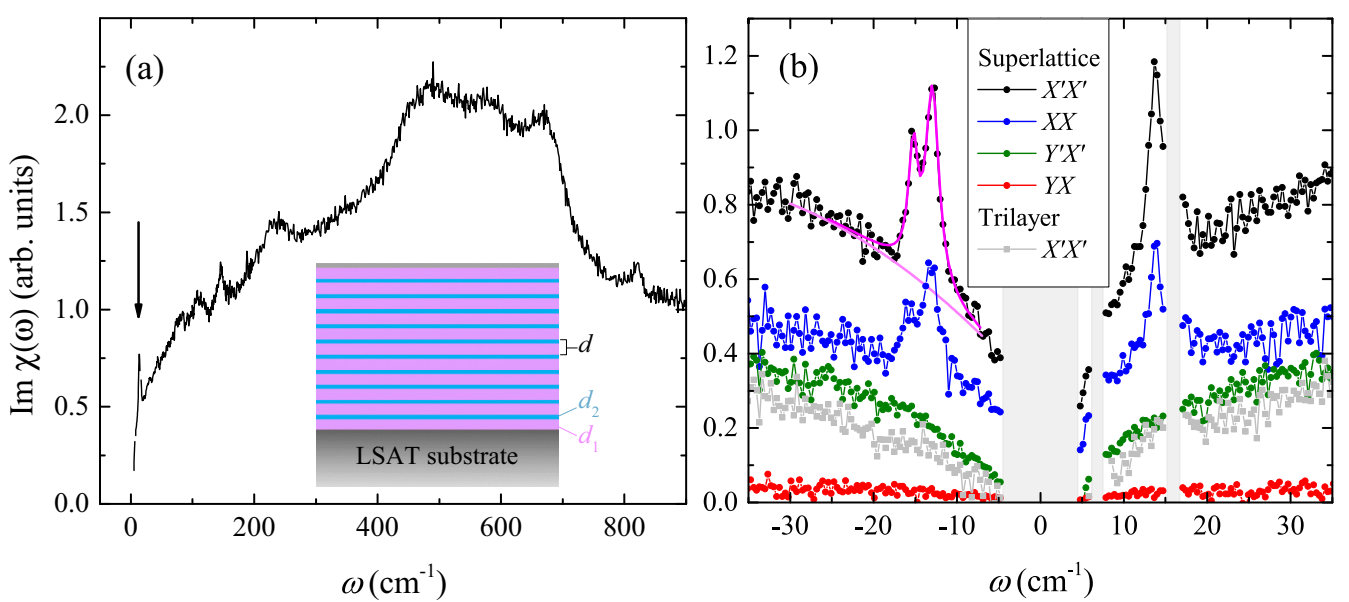

FIG. 1. (a) Spectrum of a $\operatorname{PLCMO}(10 \mathrm{~nm}) / \mathrm{YBCO}(7 \mathrm{~nm})$ superlattice, illustrated bottom center, in $z\left(X^{\prime} X^{\prime}\right) z$ scattering geometry showing the comparatively intense and narrow line width of the low-frequency modes compared with the phonon modes above $70 \mathrm{~cm}^{-1}$. (b) Polarization analysis of the low-frequency modes. The thick magenta line for the $z\left(X^{\prime} X^{\prime}\right) z$ superlattice spectrum shows a fit to the data with the modeled background as the thin magenta line. The gray data (offset by -0.5 for clarity) are for a trilayer sample of the same composition which indicates that the low-energy modes are inherent to the superlattice samples only.

grown by $\mathrm{PLD}$ on $\mathrm{La}_{0.3} \mathrm{Sr}_{0.7} \mathrm{Al}_{0.65} \mathrm{Ta}_{0.35} \mathrm{O}_{3}$ (LSAT) substrates that are $\left(\begin{array}{lll}0 & 0 & 1\end{array}\right)$ oriented following the process described in Refs. [15] and [31]. The superlattices involve 10 repetitions of cuprate-manganite layers, whose thickness we denote using the following scheme; $\operatorname{PLCMO}(10 \mathrm{~nm}) / \mathrm{YBCO}(7 \mathrm{~nm})$ translates to a 7-nm-thick YBCO layer grown on top of a 10 -nm-thick PLCMO layer. The topmost layer is the manganite, followed by a 2-nm-thick $\mathrm{LaAlO}_{3}$ capping layer to protect the film surface from degradation.

We performed ex situ studies with x-ray diffraction, x-ray reflectivity, and polarized neutron reflectivity to measure the layer thickness, uniformity, and interface roughness. Representative results can be found in the Supplementary Materials of Refs. [9] and [15]. These show that our samples are of a high quality, with a small interface roughness $(\sim 0.5 \mathrm{~nm})$ that tends to increase with additional cuprate/manganite layers and minimal chemical diffusion across the interface.

The Raman spectra were recorded with a Jobin-Yvon LabRam HR800 spectrometer using the 632.8-nm excitation line of a HeNe laser [32]. The measurements were carried out in full backscattering with geometry indicated by Porto's notation. For example, $z\left(Y^{\prime} X^{\prime}\right) z$ indicates backscattering with incident polarization $45^{\circ}$ to the $\mathrm{Mn}-\mathrm{O}$ nearest-neighbor bond with the cross-polarized scattered light measured. We find spurious reflections in our spectrometer that lead to artifacts around 7 and $15.8 \mathrm{~cm}^{-1}$; these spectral regions are removed from the reported spectra for clarity. Unless noted otherwise, the spectra shown were recorded at room temperature. The 1800 lines $/ \mathrm{mm}$ gratings give a spectral resolution of $0.3 \mathrm{~cm}^{-1}$. The laser was focused with a $\times 100$ long-working distance objective lens with a short depth of focus, NA $=0.6$, which was positioned with an accuracy of $0.5 \mu \mathrm{m}$ such that the focus is centered on the film [32]. In order to further minimize the signal from the LSAT substrate we use a 50- $\mu \mathrm{m}$ confocal hole along the scattered light path. The penetration depth of the laser light in our samples is about $70 \mathrm{~nm}$. As such, the residual substrate contribution is small, but nevertheless it is subtracted from the spectra using reference measurements for which the beam focus was moved into the substrate (see Fig. S1 of the Supplementary Material [30]). All reported spectra have been divided by the Bose thermal factors to obtain the imaginary part of the Raman scattering susceptibility, $\operatorname{Im} \chi(\omega)$. Further details and exemplary raw spectra of the superlattices and substrates can be found in the Supplementary Material [30].

\section{RESULTS}

\section{A. Exemplary case}

To set the scene, Fig. 1(a) shows the Raman Stokes signal from a PLCMO $(10 \mathrm{~nm}) / \mathrm{YBCO}(7 \mathrm{~nm})$ superlattice (sketched) collected at room temperature in $z\left(X^{\prime} X^{\prime}\right) z$ geometry over a wide spectral range. The spectrum reveals multiple broad overlapping phonon modes above $50 \mathrm{~cm}^{-1}$ arising from both YBCO, manganite, and the interaction between them. A rich spectral fingerprint is expected, as a simplified space group of the manganite, Pmma, allows 21 Raman-active modes [33] (the more realistic $P 21 / m$ symmetry, which includes the $\mathrm{MnO}_{6}$ octahedral tilts, having 54 Raman-active phonon modes). The manganite spectra are also consistent with a disordered rhombohedral phase with space group $R \bar{c} 3$ [34]. YBCO has five main Raman-active phonon modes, with additional modes in special cases of charge and oxygen ordering [35]. An analysis of this spectral region will be presented elsewhere, as here we focus on Raman scattering below $30 \mathrm{~cm}^{-1}$.

In particular, we focus on two prominent features in the low-energy spectra, which are marked by the arrow in Fig. 1(a) and highlighted in Fig. 1(b). In Fig. 1(b), both the Stokes and the anti-Stokes signals at $T=300 \mathrm{~K}$ in a narrow spectral region around the elastic line are shown. The modes are clearly pronounced for both the $z(X X) z$ and the $z\left(X^{\prime} X^{\prime}\right) z$ geometries, but they are not observed for the cross-polarized geometries.

Importantly, these sharp modes are only observed in superlattice samples. For example, we include in Fig. 1(b) 
a spectrum from a trilayer of the same material, PLCMO $(20 \mathrm{~nm}) / \mathrm{YBCO}(7 \mathrm{~nm}) / \operatorname{PLCMO}(20 \mathrm{~nm})$, in which the sharp low-energy features are absent.

To quantitatively characterize these peaks, we fit them using a quadratic background and a pseduo-Voigt line shape as detailed in the Supplementary Material [30]. An exemplary fit is represented by the magenta line superimposed on the $z\left(X^{\prime} X^{\prime}\right) z$ superlattice data in Fig. 1(b), whereas the modeled background is a lighter-colored thin magenta line. The fitted peaks shown in Fig. 1(b) are centered at $\omega_{0}=-12.9$ and $-15.2 \mathrm{~cm}^{-1}$, with the absolute uncertainty in the peak positions estimated to be $0.3 \mathrm{~cm}^{-1}$, primarily due to systematic uncertainties. The half-widths at half-maximum (HWHM) are $\gamma=1.0$ and $0.5 \mathrm{~cm}^{-1}$ respectively. The area of each mode, $S W$, is proportional to its Raman susceptibility. While we cannot quantify the Raman susceptibility from our data in absolute terms, we can compare the Bose-corrected areas of these new modes with that of a regular phonon mode. In particular, we find an area of $S W \approx 0.2$ (a.u.) for the phonon excitation at $\omega_{0} \approx 145 \mathrm{~cm}^{-1}$ and areas of $S W=0.5$ and 0.3 for the two low-energy modes, respectively. This illustrates that the new modes have Raman cross sections comparable to the weaker phonon modes above $70 \mathrm{~cm}^{-1}$.

In transition-metal oxides like those studied here, modes in this frequency range might be ascribed to magnetic excitations [36]. However, for several reasons this is unlikely in our case, despite the significant Mn magnetic moments. First, longrange magnetic order is established only below $T \approx 140 \mathrm{~K}$ in our PLCMO and NCSMO samples [9,15,37], whereas these peaks are intense and sharp already at room temperature. Second, the new modes are only observed for superlattice samples, and not in films of the pure manganite material with a thickness comparable to that of the superlattices. Third, whereas magnons are usually observed in crossed polarization, the $z(X Y) z$ and $z\left(X^{\prime} Y^{\prime}\right) z$ polarization channels of our superlattices do not exhibit low-energy modes [Fig. 1(b)]. In addition, the low-energy modes are not observed in trilayer samples, which rules out that they originate solely due to an interaction between the cuprate and the manganite.

\section{B. Sample dependence}

Furthermore, Fig. 2 illustrates that the position of these peaks depends on the bilayer thickness in the superlattice samples (i.e., the sum of the YBCO and manganite film thicknesses). Figure 2(a) shows $z\left(X^{\prime} X^{\prime}\right) z$ spectra for superlattice samples with a range of bilayer thicknesses, $d$. Spectra have been offset for clarity. The shift of the modes to lower energies with larger bilayer thicknesses is clearly seen from the raw data. Figure 2(b) shows the fitted energies of the two observable low-energy modes, $\omega_{0}$, as data points plotted vs $d^{-1}$. The solid line in the figure has the form $\omega_{0}=v_{s} d^{-1}$, where the meaning and choice of the value $v_{s}$ are discussed below. The peak areas, $S W$, and widths, $\gamma$, do not appear to show any systematic variation across the samples studied. Finally, we note the possible presence of additional lowenergy peaks barely resolved by our measurements, except in particular cases such as the $d=16.5 \mathrm{~nm}$ sample (brown curve).

These observations show that the low-energy modes probably arise from a backfolding of the Brillouin zone, due to
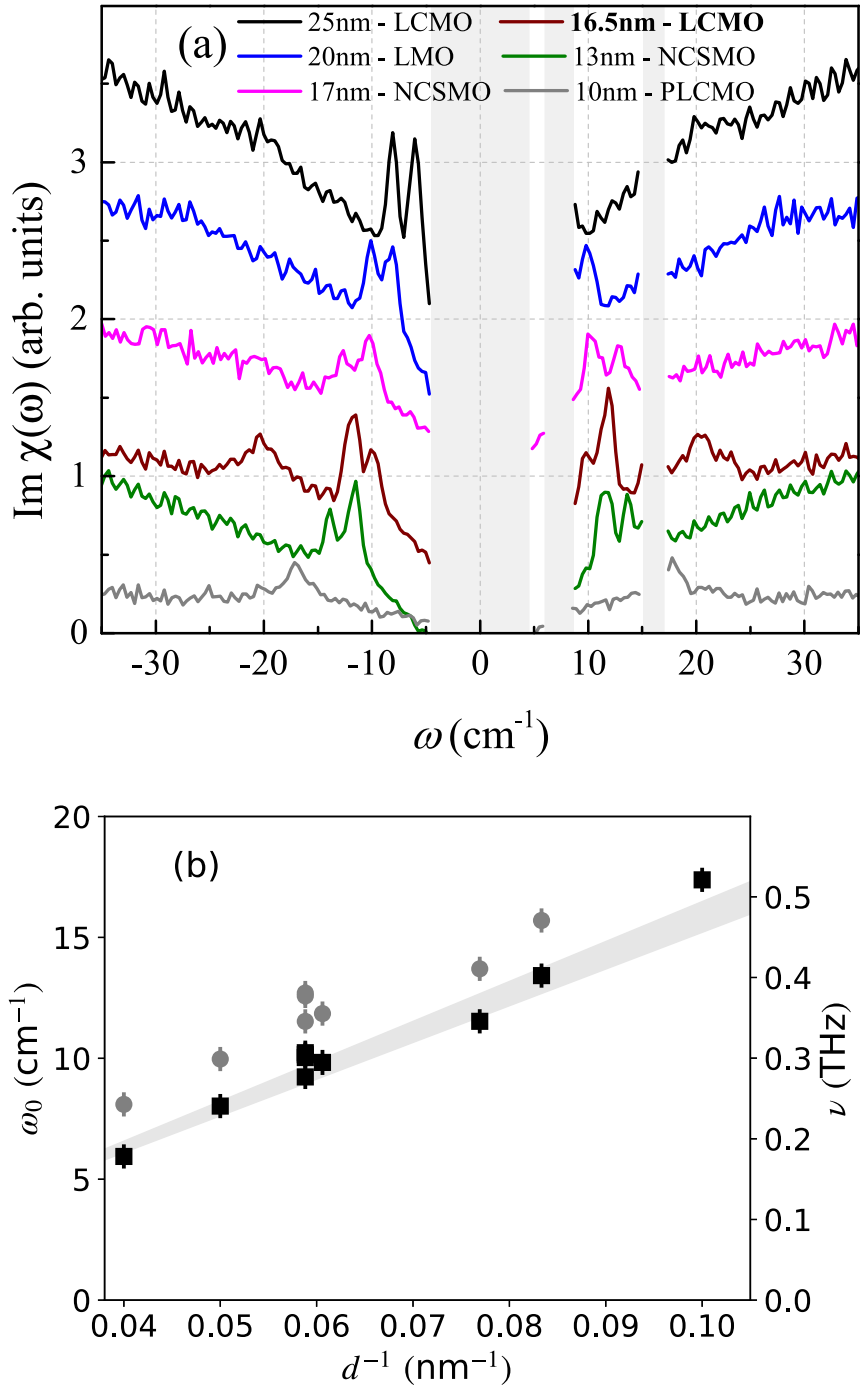

FIG. 2. (a) Spectra from multiple superlattice samples with bilayer thickness, $d$, and the manganite compositions indicated in the legend. (b) Center positions of the two observable low-energy modes versus $d^{-1}$. The shaded line represents the expected average position of the two low-frequency modes based on the speed of sound in the superlattice.

the superlattice periodicity, which brings new Raman-active excitations at low energies onto the $\Gamma$ point.

Such a situation has been well documented and analyzed in superlattices comprised of GaAs-AlAs and other semiconductors for acoustic phonon branches [22,24-26,28]. There are well-established models for this situation, starting from either a continuum approximation or linear-chain type models $[24,27,28]$, of which detailed versions have been developed in order to capture finite-size effects of the sample [29]. All such models agree, however, with the general behavior captured by the simpler Rytov model [38]. Within this model, the phonon dispersion is described by

$$
\begin{aligned}
\cos (q d)= & \cos \left(\frac{\omega d_{1}}{v_{s, 1}}\right) \cos \left(\frac{\omega d_{2}}{v_{s, 2}}\right) \\
& -\frac{1+\kappa^{2}}{2 \kappa} \sin \left(\frac{\omega d_{1}}{v_{s, 1}}\right) \sin \left(\frac{\omega d_{2}}{v_{s, 2}}\right),
\end{aligned}
$$



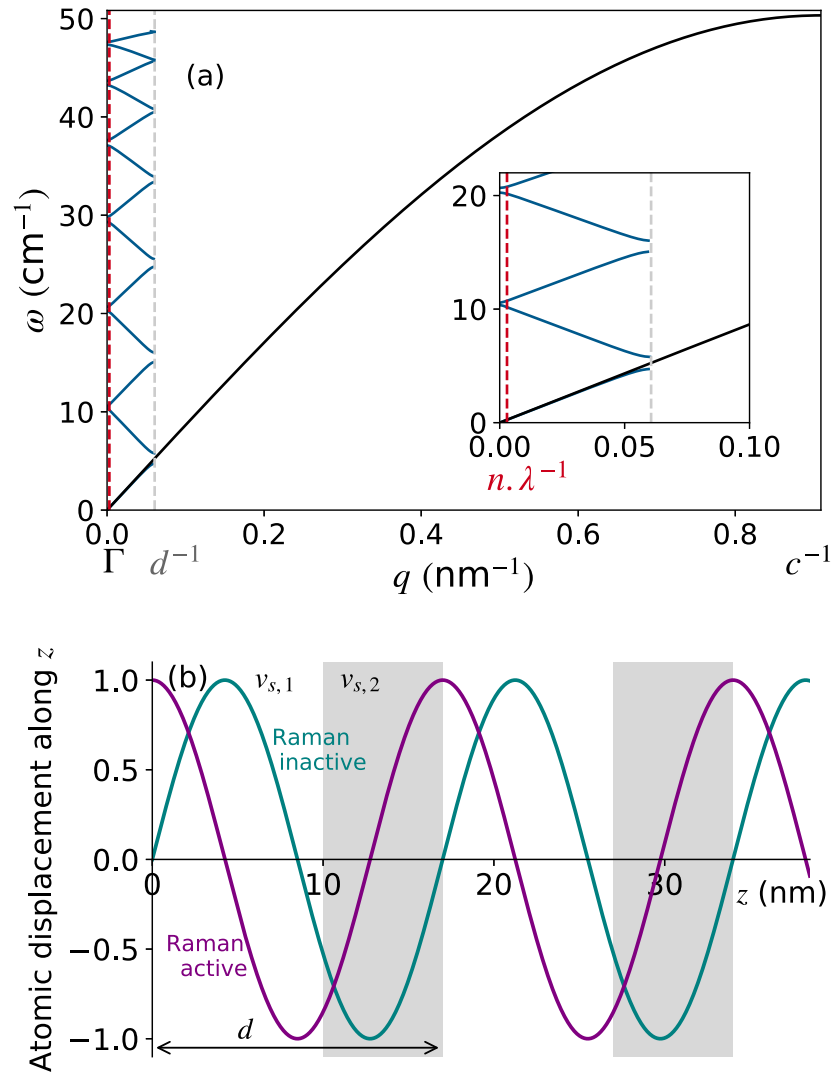

FIG. 3. (a) Sketch of phonon backfolding with approximate values for our samples. The black line shows a typical dispersion of an acoustic phonon branch. The additional periodicity of the superlattice, $d$, backfolds the phonon branch around $d^{-1}$. There is a splitting of the phonon branches near $q=0$ and $d^{-1}$ creating phonon bandgaps there. The Raman experiment probes the phonon modes with $q=n \lambda^{-1}$, shown by the dashed red line. (b) Sketch of the amplitude of atomic displacement for the two first-order backfolded modes at $\Gamma$. Shaded regions represent the material with the higher speed of sound, $v_{s, 2}>v_{s, 1}$.

where the subscripts 1 and 2 denote the two materials in the superlattice and $v_{s}=\sqrt{c_{33} / \rho}$ is the sound velocity (with $\rho$ the density of the material and $c_{33}$ the elastic modulus along the $c$-axis direction ). $d_{1}$ and $d_{2}$ are the thicknesses of the layers comprising the superlattice and $\kappa \equiv v_{s, 2} \rho_{2} / v_{s, 1} \rho_{1}$. This expression describes a folding of the phonon dispersions about $d^{-1}$, where $d=d_{1}+d_{2}$, and the opening of gaps at the zone center and boundaries. Figure 3(a) sketches the backfolding effect with approximate values for our experiments. With the Raman measurements, we (de)excite the phonon modes at $q=n \lambda^{-1}$, where $n$ is the refractive index of the superlattice and $\lambda$ the laser wavelength.

This model describes our observations accurately. In particular, the straight line in Fig. 2(b) is given by $v_{s} d^{-1}$, where $d$ is obtained from the nominal layer thicknesses (estimated from $\mathrm{X}$-ray reflectivity measurements) and $v_{s}=$ $\frac{7}{17} v_{s, \mathrm{YBCO}}+\frac{10}{17} v_{s, \text { manganite }}$ is the weighted average of the $c$ axis speed of sound in the two materials (here we are using the most common 7-nm YBCO layer and 10-nm manganite layer thicknesses) [24]. The value of $v_{s}$ is $4750 \pm 200 \mathrm{~m} \mathrm{~s}^{-1}$ as determined from the bulk moduli and densities $[39,40]$, but similar values are obtained from other measurements of the speed of sound [41-44]. The width of the line in Fig. 2(b) comes from the uncertainty in $v_{s}$ for the individual YBCO and manganite layers. $v_{s} d^{-1}$ represents an average of the two peak positions, which are split due to mixing and the finite $q$ of the laser line [27]. Therefore, our experimental results are consistent with the slightly higher value of $v_{s} \approx 5150 \mathrm{~m} \mathrm{~s}^{-1}$

A generalized relaxation time of the mode can be expressed as $\tau=\gamma^{-1}$, which is between 30 and $60 \mathrm{ps,} \mathrm{depending}$ on the mode. If we take $\tau=40 \mathrm{ps}$ and $v_{s}=5150 \mathrm{~m} \mathrm{~s}^{-1}$, then the scattering length of the mode is $l=v_{s} \tau \approx 200 \mathrm{~nm}$. This is close to the total film thickness and suggestive of dissipative scattering of the phonon at the top surface of the superlattice and bottom interface with the substrate rather than the $\mathrm{YBCO} /$ manganite interfaces. Similar conclusions were made by Li et al. from pump-probe reflection measurements of superlattices with two different bilayer thicknesses [42]. This conclusion is also supported by the observation that the ratio of the mode width to the resonant frequency, $\gamma / \omega_{0}$, is approximately 0.07 and much higher than the ratio of $\sim 10^{-3}$ that is measured for acoustic modes at room temperature by resonant ultrasound spectroscopy on single crystals of YBCO [45] or manganites [46]; i.e. the phonon scattering is enhanced in the superlattices with respect to the bulk crystals. On the other hand, we cannot rule out alternate explanations for the shortened phonon lifetime that we infer from the phonon linewidth, such as from inhomogeneity across the beam spot.

We now discuss the intensity of the peaks. Close to the Brillouin-zone center, the phonon mode upper and lower branches of the backfolded dispersion have Raman-active and Raman-inactive symmetries. The atomic displacement amplitude for the modes, that is, the two first-order backfolded modes phonon modes at the Brillouin-zone center, are sketched in Fig. 3(b) following Ref. [22]. One mode is a symmetric stretch of the bilayer unit that causes a change in polarizability and is hence expected to be Raman active. This mode has maximal amplitude at $z=0$ and is represented by the purple curve in Fig. 3(b). The other mode is an antisymmetric stretch and is not expected to be Raman active (offresonance). We note that the finite $q$ of phonon branches that we measure in backscattering geometry leads to a mixing of the two symmetries $[22,47,48]$, but we still expect the relative Raman cross section of the two branches to be different in our experiments.

The relative thickness and speed of sound of the manganite and YBCO layers determine whether the upper or lower branch has dominant Raman-active symmetry. We denote these $d_{1}$ and $d_{2}$, respectively, whereby layer 1 (the manganite) has a slower speed of sound than layer 2 (YBCO): $v_{s, 1}<v_{s, 2}$. For $d_{1}<d_{2}$, the Raman-inactive mode has a lower energy than the Raman-active mode, and this results in the lowerenergy mode having a lower intensity in the spectra. The sample representing this case is shown in boldface in the legend of Fig. 2(a). For most of our samples the $d_{1}>d_{2}$ condition instead holds so that the situation is reversed, with the higher-energy mode having the lower intensity. For $d_{1}=$ $d_{2}$, the second-order backfolded phonon branch will not be Raman active, and this is the case for the 20-nm LMO sample in Fig. 2(a). 
However, for all samples we barely see the second-order modes. We are not certain of the reason for this, but it could be an indication of interface roughness, as we note that in GaAs/AlAs superlattices the intensity of the backfolded modes was observed to decrease upon annealing of the sample at $850^{\circ} \mathrm{C}$, a process that introduces interface roughness by diffusion of the ions across the interface [27]. The $d=16.5 \mathrm{~nm}$ sample (brown curve) is a possible exception, where there is a clear peak at the expected position for the second-order backfolded modes. Here we also note that backfolding of the acoustic phonon branch was seen by terahertz/infrared spectroscopy of bulk manganites due to the additional periodicity of the charge-ordered state [49], as well as a disorder-activated "boson peak" at approximately $20 \mathrm{~cm}^{-1}$ and temperatures below $120 \mathrm{~K}$. We do not believe this to be a plausible origin of the $20 \mathrm{~cm}^{-1}$ peak in our data that were obtained at room temperature. More quantitative predictions of the phonon intensities require first-principles calculations of the photoelastic coefficients [27,47].

Finally we note that we observe similar low-frequency modes in superlattices grown in our laboratory where the YBCO is replaced with another metal oxide. These include $\mathrm{SrFeO}_{3} / \mathrm{La}_{2 / 3} \mathrm{Ca}_{1 / 3} \mathrm{MnO}_{3}$ [50] and $\mathrm{SrRuO}_{3} / \mathrm{La}_{2 / 3} \mathrm{Ca}_{1 / 3} \mathrm{MnO}_{3}$ superlattices.

\section{Spatial dependence}

The results above show that these low-energy modes can be used to determine the spatial variation in a superlattice's bilayer thickness (or more generally its repeat-unit thickness), $d$, because (i) their position, $\omega_{0}$, is a function of $d$ and (ii) the micro-Raman technique we use here has a $10-\mu \mathrm{m}^{2} \mathrm{spa}-$ tial resolution in the plane. Spatial uniformity is of particular interest for growth techniques wherein the deposition rate of the films may not be constant across the sample. One particularly important example in terms of oxide materials is the growth by PLD on substrates with a surface area comparable to the size of the plasma plume. The spatial dependence of the intensity and HWHM may also be used to characterize the film quality.

To exemplify the potential of our method, we performed measurements on a $7 \times 7$-point grid across the surface of a nominally $\mathrm{LCMO}(10 \mathrm{~nm}) / \mathrm{YBCO}(10 \mathrm{~nm})$ superlattice grown by PLD on an LSAT substrate with surface area $10 \times 10 \mathrm{~mm}^{2}$. The results are summarized in Fig. 4. The color scale represents $d$ as determined from the fitted $\omega_{0}$ of the two low-energy modes at each point and $v_{s}=5000 \mathrm{~m} \mathrm{~s}^{-1}$ as determined in Fig. 2(b). The data are linearly interpolated between the measurement points and we estimate the uncertainty in $d$ to be $\sim 1$ $\mathrm{nm}$. Figure 4 shows that the LCMO-YBCO layer thicknesses are not uniform across the approximate $9 \times 9 \mathrm{~mm}^{2}$ area that was measured. Instead, we identify a smooth gradient in the value of $d$ from one corner to the opposite corner.

\section{Temperature dependence}

The multilayers studied here exhibit a range of electronic and magnetic phase transitions at temperatures below $300 \mathrm{~K}[9,15,37,51,52]$ and the materials are known for significant electron-phonon and spin-lattice coupling $[4,5,7,13]$. As such, we investigated the temperature dependence of the

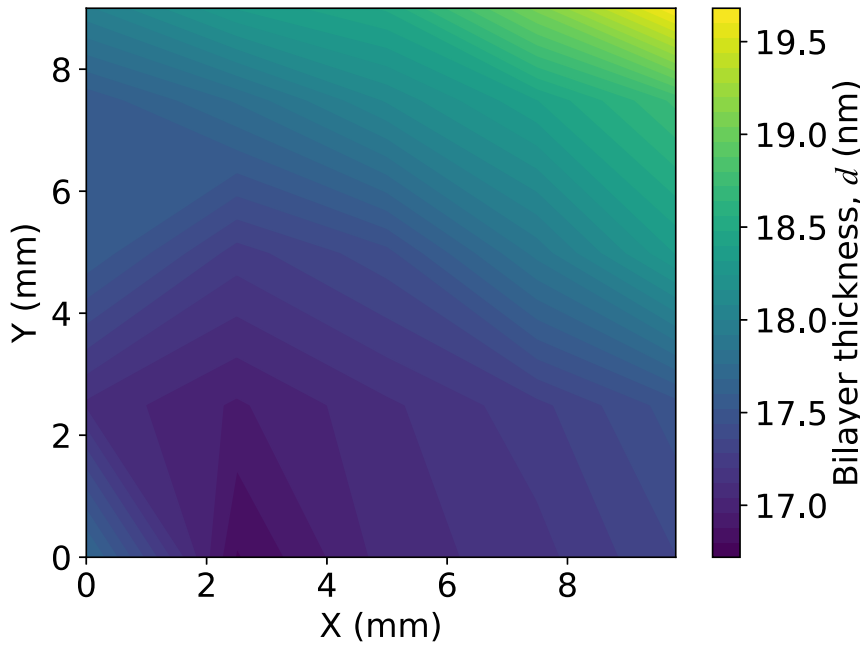

FIG. 4. A contour plot of the combined thickness of the LCMO and YBCO layers, $d$, determined from $\omega_{0}$ of the low-frequency modes. There is a smooth gradient in $d$ from one corner to the opposite.

backfolded phonon modes. Figure 5(a) shows the Stokes signal for a $\operatorname{NCSMO}(10 \mathrm{~nm}) / \mathrm{YBCO}(7 \mathrm{~nm})$ superlattice with nominal $x=0.5$ and $y=0.33$ for NCSMO between room temperature and a nominal temperature of $10 \mathrm{~K}$. We find only a subtle temperature dependence, except for a possible increase in intensity below $50 \mathrm{~K}$ (the Bose thermal correction at low energies is particularly large and, hence, sensitive to imperfect background signal subtraction and laser-heating effects). Qualitatively similar temperature dependencies were observed for other superlattices, where measured.

Figures $5(\mathrm{~b})-5(\mathrm{~d})$ show the results of fitting the Stokes signal from the lower-energy mode. We note here that first the background was subtracted, then the Bose thermal factor correction applied, before fitting the peaks. This procedure was adopted to reduce the uncertainty of the background contribution to the peak intensity. The $\omega_{0}$ of the mode shows only slight variation across the whole temperature range. In absolute terms, the variation is comparable to the absolute uncertainty in $\omega$ of $0.3 \mathrm{~cm}^{-1}$ that we estimate, as shown in Fig. 5(b). Figure 5(c) shows the temperature dependence of the fitted peak area, $S W . S W$ is proportional to the Raman cross section and shows an increase at low temperatures. From the Stokes/anti-Stokes ratio of the low-frequency modes we have obtained laser-heating estimates of up to $40 \mathrm{~K}$ at base temperature (which is nominally $10 \mathrm{~K}$ ) following the method of Herman [53], although the uncertainty/scatter in such estimates is of the order of $50 \mathrm{~K}$. A $40 \mathrm{~K}$ laser-heating effect would be enough to compensate the low-temperature increase in $S W$ and recover the customary temperature-independent $S W$, and we cannot yet rule this out as an explanation for the increased peak area below the nominal temperature of $50 \mathrm{~K}$. Figure 5(d) shows that there is no observable temperature dependence of the HWHM, suggesting no significant additional relaxation/scattering channels for the phonons open in the various magnetic, charge, and superconducting ordered phases at lower temperatures. 

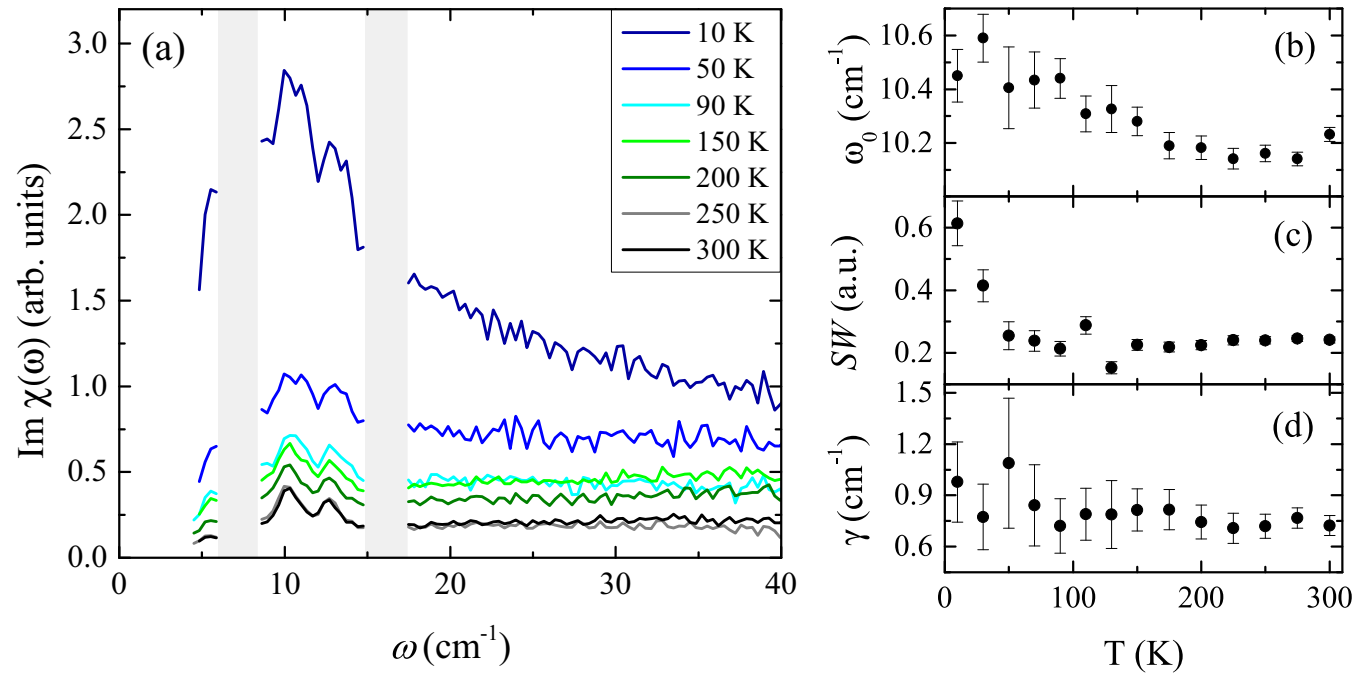

FIG. 5. (a) Representative temperature dependence of the low-frequency spectra in $z(X X) z$ geometry, from a NCSMO(10 nm)/YBCO $(7 \mathrm{~nm}$ ) superlattice. (b) Fitted center position, (c) intensity, and (d) HWHM of the lower-energy peak.

\section{DISCUSSION AND CONCLUSIONS}

These results demonstrate the remarkable quality of transition-metal oxide films that can now be grown with pulsed laser deposition. The experimental methodology we used to characterize the superlattices is nondestructive, relatively accessible, and rapid.

There are several ways in which future measurements could be improved to gain a richer characterization of the superlattice film quality. First, higher-resolution spectra would better resolve the peak positions, intensity, widths, and asymmetry. Such higher-resolution spectra would be desirable for low-temperature studies of these materials in order to study the coupling between this phonon branch and the spin or lowenergy electronic systems. Furthermore, it may be possible to observe the fine structure of these peaks, which would allow an analysis beyond the continuum Rytov model, for example, using finite-size, linear-chain-type models that can model thickness variations in the superlattice [29]. Second, it would be possible to independently determine both $d$ and $v_{s}$ from the Raman data alone, with measurements using multiple laser wavelengths. Such measurements, or the more challenging forward-scattering geometry measurements [47,48], would also reveal in more detail the splitting of the two phonon branches that is caused by interatomic coupling between the two metal-oxide layers [27].

In summary, we report systematic measurements of lowfrequency modes in metal-oxide superlattices grown by pulsed laser deposition. We show that these modes arise from a backfolded $c$-axis acoustic phonon branch. As such, their observation demonstrates the high quality of the thin-film superlattices. The modes can be used to characterize the bilayer thickness of the superlattice and/or the $c$-axis speed of sound. We utilized the spatial resolution of the Raman microscopy to map the film thickness inhomogeneity across a larger, $10 \times 10 \mathrm{~mm}$ sample. This information is important for monitoring and improving the quality of future metal-oxide superlattices which might comprise the building blocks of next-generation electronic devices.

\section{ACKNOWLEDGMENTS}

We thank Dr. F. Weber and Dr. R. Heid for valuable discussions on this work. The work was supported by the Schweizerische Nationalfonds (SNF) through Grant No. 200020172611. B.P.P.M. acknowledges support from the Rutherford Foundation of New Zealand. A.C. acknowledges support from the MacDiarmid Institute.
[1] J. Chakhalian, J. W. Freeland, H.-U. Habermeier, G. Cristiani, G. Khaliullin, M. van Veenendaal, and B. Keimer, Science 318, 1114 (2007).

[2] N. Reyren, S. Thiel, A. D. Caviglia, L. F. Kourkoutis, G. Hammerl, C. Richter, C. W. Schneider, T. Kopp, A.-S. Ruetschi, D. Jaccard, M. Gabay, D. A. Muller, J.-M. Triscone, and J. Mannhart, Science 317, 1196 (2007).

[3] M. Bibes, J. E. Villegas, and A. Barthélémy, Adv. Phys. 60, 5 (2011).

[4] H. Y. Hwang, Y. Iwasa, M. Kawasaki, B. Keimer, N. Nagaosa, and Y. Tokura, Nat. Mater. 11, 103 (2012).

[5] N. Driza, S. Blanco-Canosa, M. Bakr, S. Soltan, M. Khalid, L. Mustafa, K. Kawashima, G. Christiani, H.-U. Habermeier, G.
Khaliullin, C. Ulrich, M. Le Tacon, and B. Keimer, Nat. Mater. 11, 675 (2012).

[6] K. Rogdakis, J. Seo, Z. Viskadourakis, Y. Wang, L. A. Qune, E. Choi, J. Burton, E. Tsymbal, J. Lee, and C. Panagopoulos, Nat. Commun. 3, 1064 (2012).

[7] J. Chakhalian, J. W. Freeland, A. J. Millis, C. Panagopoulos, and J. M. Rondinelli, Rev. Mod. Phys. 86, 1189 (2014).

[8] M. Lorenz, M. R. Rao, T. Venkatesan, E. Fortunato, P. Barquinha, R. Branquinho, D. Salgueiro, R. Martins, E. Carlos, A. Liu et al., J. Phys. D 49, 433001 (2016).

[9] B. P. P. Mallett, J. Khmaladze, P. Marsik, E. Perret, A. Cerreta, M. Orlita, N. Biškup, M. Varela, and C. Bernhard, Phys. Rev. B 94, 180503(R) (2016). 
[10] M. Keunecke, F. Lyzwa, D. Schwarzbach, V. Roddatis, N. Gauquelin, K. Müller-Caspary, J. Verbeeck, S. J. Callori, F. Klose, M. Jungbauer et al., Adv. Funct. Mater. 2019, 1808270 (2019).

[11] D. L. Smith and C. Mailhiot, Rev. Mod. Phys. 62, 173 (1990).

[12] J. Mannhart and D. G. Schlom, Science 327, 1607 (2010).

[13] Y. Tokura, Rep. Prog. Phys. 69, 797 (2006).

[14] B. Keimer, S. Kivelson, M. Norman, S. Uchida, and J. Zaanen, Nature 518, 179 (2015).

[15] E. Perret, C. Monney, S. Johnston, J. Khmaladze, F. Lyzwa, R. Gaina, M. Dantz, J. Pelliciari, C. Piamonteze, B. Mallett, M. Minola, B. Keimer, T. Schmitt, and C. Bernhard, Commun. Phys. 1, 45 (2018).

[16] J. Ravichandran, A. K. Yadav, R. Cheaito, P. B. Rossen, A. Soukiassian, S. Suresha, J. C. Duda, B. M. Foley, C.-H. Lee, Y. Zhu et al., Nat. Mater. 13, 168 (2014).

[17] M. Maldovan, Nat. Mater. 14, 667 (2015).

[18] D. P. Norton, Mater. Sci. Eng. R 43, 139 (2004).

[19] D. Dijkkamp, T. Venkatesan, X. Wu, S. Shaheen, N. Jisrawi, Y. Min-Lee, W. McLean, and M. Croft, Appl. Phys. Lett. 51, 619 (1987).

[20] D. Schlom, J. N. Eckstein, E. Hellman, S. Streiffer, J. Harris Jr., M. Beasley, J. Bravman, T. Geballe, C. Webb, K. Von Dessonneck et al., Appl. Phys. Lett. 53, 1660 (1988).

[21] R. Rao, Q. Gan, C. Eom, Y. Suzuki, A. McDaniel, and J. Hsu, Appl. Phys. Lett. 69, 3911 (1996).

[22] C. Colvard, R. Merlin, M. V. Klein, and A. C. Gossard, Phys. Rev. Lett. 45, 298 (1980).

[23] B. Jusserand, D. Paquet, and F. Mollot, Phys. Rev. Lett. 63, 2397 (1989).

[24] M. Cardona, Superlattices Microstruct. 5, 27 (1989).

[25] B. D. Rouhani and E. Khourdifi, Light Scattering in Semiconductor Structures and Superlattices (Springer, New York, 1991), pp. 139-158.

[26] J. Sapriel and J. He, Light Scattering in Semiconductor Structures and Superlattices (Springer, New York, 1991), pp. 123138.

[27] C. Colvard, T. A. Gant, M. V. Klein, R. Merlin, R. Fischer, H. Morkoc, and A. C. Gossard, Phys. Rev. B 31, 2080 (1985).

[28] B. Jusserand and M. Cardona, Top. Appl. Phys. 66, 49 (1989).

[29] M. W. C. Dharma-wardana, P. X. Zhang, and D. J. Lockwood, Phys. Rev. B 48, 11960 (1993).

[30] See Supplemental Material at http://link.aps.org/supplemental/ 10.1103/PhysRevMaterials.4.043606 for further details on data analysis methods and the samples studied. Exemplary raw data are shown and data on other metal oxide superlattices.

[31] V. K. Malik, I. Marozau, S. Das, B. Doggett, D. K. Satapathy, M. A. Uribe-Laverde, N. Biskup, M. Varela, C. W. Schneider, C. Marcelot, J. Stahn, and C. Bernhard, Phys. Rev. B 85, 054514 (2012).

[32] M. Hepting, M. Minola, A. Frano, G. Cristiani, G. Logvenov, E. Schierle, M. Wu, M. Bluschke, E. Weschke, H.-U. Habermeier, E. Benckiser, M. Le Tacon, and B. Keimer, Phys. Rev. Lett. 113, 227206 (2014).

[33] M. V. Abrashev, J. Bäckström, L. Börjesson, M. Pissas, N. Kolev, and M. N. Iliev, Phys. Rev. B 64, 144429 (2001).
[34] M. N. Iliev, M. V. Abrashev, V. N. Popov, and V. G. Hadjiev, Phys. Rev. B 67, 212301 (2003).

[35] M. Bakr, S. M. Souliou, S. Blanco-Canosa, I. Zegkinoglou, H. Gretarsson, J. Strempfer, T. Loew, C. T. Lin, R. Liang, D. A. Bonn, W. N. Hardy, B. Keimer, and M. Le Tacon, Phys. Rev. B 88, 214517 (2013).

[36] P. Murugavel, C. Narayana, A. Sood, S. Parashar, A. Raju, and C. Rao, Europhys. Lett. 52, 461 (2000).

[37] J. Khmaladze, S. Sarkar, M. Soulier, F. Lyzwa, R. de Andres Prada, E. Perret, B. P. P. Mallett, M. Minola, B. Keimer, and C. Bernhard, Phys. Rev. Mater. 3, 084801 (2019).

[38] S. Rytov, Akusticheskii Zhurnal 2, 71 (1956) [Sov. Phys. Acoust. 2, 68 (1956)].

[39] H. Hazama, Y. Nemoto, T. Goto, A. Asamitsu, and Y. Tokura, Physica B: Condens. Matter 281-282, 487 (2000).

[40] J. Jorgensen, S. Pei, P. Lightfoot, D. Hinks, B. Veal, B. Dabrowski, A. Paulikas, R. Kleb, and I. Brown, Physica C 171, 93 (1990).

[41] M. Seikh, C. Narayana, S. Parashar, and A. Sood, Solid State Commun. 127, 209 (2003).

[42] W. Li, B. He, C. Zhang, S. Liu, X. Liu, S. Middey, J. Chakhalian, X. Wang, and M. Xiao, Appl. Phys. Lett. 108, 132601 (2016).

[43] L. Pintschovius and W. Reichardt, in Neutron Scattering in Layered Copper-Oxide Superconductors, edited by A. Furrer (Springer, Dordrecht, Netherlands, 1998), pp. 165-223.

[44] F. Weber, S. Rosenkranz, J.-P. Castellan, R. Osborn, H. Zheng, J. F. Mitchell, Y. Chen, S. Chi, J. W. Lynn, and D. Reznik, Phys. Rev. Lett. 107, 207202 (2011).

[45] A. Shekhter, B. Ramshaw, R. Liang, W. Hardy, D. Bonn, F. F Balakirev, R. D. McDonald, J. B. Betts, S. C. Riggs, and A. Migliori, Nature 498, 75 (2013).

[46] M. A. Carpenter, C. J. Howard, R. E. A. McKnight, A. Migliori, J. B. Betts, and V. R. Fanelli, Phys. Rev. B 82, 134123 (2010).

[47] B. Jusserand, F. Alexandre, J. Dubard, and D. Paquet, Phys. Rev. B 33, 2897 (1986).

[48] B. Jusserand, D. Paquet, F. Mollot, F. Alexandre, and G. Le Roux, Phys. Rev. B 35, 2808 (1987).

[49] B. Gorshunov, E. Zhukova, V. I. Torgashev, L. S. Kadyrov, E. A. Motovilova, F. Fischgrabe, V. Moshnyaga, T. Zhang, R. Kremer, U. Pracht, S. Zapf, and M. Dressel, Phys. Rev. B 87, 245124 (2013).

[50] E. Perret, K. Sen, J. Khmaladze, B. P. P. Mallett, M. Yazdi-Rizi, P. Marsik, S. Das, I. Marozau, M. A. Uribe-Laverde, R. de Andrés Prada, J. Strempfer, M. Döbeli, N. Biškup, M. Varela, Y.-L. Mathis, and C. Bernhard, J. Phys.: Condens. Matter 29, 495601 (2017).

[51] T. Golod, A. Rydh, V. M. Krasnov, I. Marozau, M. A. UribeLaverde, D. K. Satapathy, T. Wagner, and C. Bernhard, Phys. Rev. B 87, 134520 (2013).

[52] K. Sen, E. Perret, A. Alberca, M. A. Uribe-Laverde, I. Marozau, M. Yazdi-Rizi, B. P. P. Mallett, P. Marsik, C. Piamonteze, Y. Khaydukov, M. Döbeli, T. Keller, N. Biškup, M. Varela, J. Vašátko, D. Munzar, and C. Bernhard, Phys. Rev. B 93, 205131 (2016).

[53] I. P. Herman, J. Appl. Phys. 109, 016103 (2011). 